\title{
ACE2: A Double-Edged Sword Against SARS CoV-2 Associated Cardiovascular Complications and Endothelial Dysfunction
}

\author{
Pratima Kumari \#* , Shaligram Sharma ${ }^{\#}$ \\ Department of Biology, College of Arts and Sciences, Georgia State University, Atlanta, GA, USA 30303 \\ \# These authors contributed equally to the work \\ *Corresponding Author
}

\begin{abstract}
The outbreak of novel Severe Acute Respiratory Syndrome Coronavirus 2 (SARS-CoV2) during late December 2019 in Wuhan, Hubei Province, China, has become a pandemic of global concern in a very short time, impacting human life and economic slowdown. The severity of SARS-CoV2 infection can be ascertained by an increased number of human deaths, specifically in older populations and patients with predisposed cardiovascular disease (CVD) complications. SARS CoV-2 binds to Angiotensin-Converting Enzyme-2 (ACE2) receptors on host cells, followed by its internalization, rapid multiplication, and instigate cytokine storm. This review aims to decipher the role of ACE2 in SARS-CoV2 infected patients with pre-existing CVD conditions. While in CVD patients, stimulation of ACE2 expression protects against CVD-associated complications through antagonizing the detrimental effects of Angiotensin II (Ang II) to maintain vascular homeostasis and production of nitric oxides in blood vessels. It is still unclear why CVD patients are at higher risk of SARS-CoV2 infection and have a higher mortality rate. Endothelial Cells (ECs) are monolayers of cells covering the inner wall of blood vessels and all major organs in our body. They play an essential role in maintaining normal vasculature; therefore, ECs dysfunction has been considered the CVD hallmark. Improvement in CVD is related to the restoration of ECs function. Exploring the role of ECs dysfunction concerning the SARS-CoV2-CVD molecular axis could help decipher why CVD patients are at increased risk of novel coronavirus-related fatalities.
\end{abstract}

Keywords: Atherosclerosis, Cardiovascular disease, SARS CoV-2, ACE-2 receptors, Endothelial dysfunction, Blood vessels

\section{INTRODUCTION}

$\mathrm{C}$ VD, including atherosclerosis, is the most common cause of death worldwide, posing severe health and economic burden [1]. Family history of coronary heart disease, high level of Low-density lipids (LDL) or lower level of high-density lipid (HDL), obesity, diabetes, and cigarette smoking are the most common risk factors associated with CVD [2, 3]. Pathobiology of atherosclerosis involves injury to endothelial lining, accumulation of lipid into the subendothelial lining in vascular smooth muscle cells, and inflammation. ECs are monolayers of cells covering the inner wall of blood vessels and are responsible for maintaining vascular homeostasis through the secretion of vasoactive substances such as NO to maintain vasculature integrity. Therefore, dysfunction of endothelial cells has been considered a prominent hallmark of cardiovascular events for decades. ECs respond to various stimuli by releasing vasoactive substances like nitric oxide (NO), carbon mono oxide (CO), endothelin, and superoxide. NO is a small gaseous molecule that diffuses into Vascular Smooth Muscle Cells (VSMCs) in blood vessels and regulates cyclic guanosine monophosphate (cGMP) production, which is required for vasodilation and subsequent relaxation of vessels [4-7]. The presence of membrane-bound receptors for growth factors, metabolites, as endothelin-1 and hormones, and surface receptors for cell-cell and cell-matrix interaction, including ACE2, make ECs a critical barrier of the vasculature. EC line all major organs in the body, such as kidneys, heart, lungs, gut, and brain, and it also expresses ACE2 cell surface receptors that the virion binds to. As a hallmark of atherosclerosis and CVD, ECs dysfunction draws our attention as a target that could be linked with COVID-19 severity. Restoration of ECs function may play an essential role in minimizing viral burden, given its critical role in the vasculature.

\section{Virus Structure and genomic organization}

The non-segmented positive-sense RNA genome of Coronaviruses $(\mathrm{CoV})$ is the largest genome among all RNA viruses with approximately $30 \mathrm{~Kb}$ in size. Spike-like structures on the outer envelope of $\mathrm{CoV}$ are a characteristic feature of the enveloped virus. The virus particle has four structural proteins, namely spike (S), membrane (M), envelope (E) and, nucleocapsid (N) proteins (Fehr and Perlman 2015). Functionally, S protein facilitates virus attachment to the host cell surface receptors and internalization of virus inside the host cell; $\mathrm{S}$ protein is the most abundant glycoprotein. $\mathrm{M}$ protein is required for virus assembly and maintains the shape of the viral envelope. Assembly and release of the virus particle require the interaction between less abundant protein $\mathrm{E}$ and $\mathrm{M}[8,9]$. According to Stohlman et al, deletion of the $\mathrm{E}$ gene attenuates the virus as the $\mathrm{E}$ gene encodes a small multifunctional protein with ion channel activity, which plays an essential role in virus-host interaction [10]. N 
protein is the sole nucleocapsid protein, which has $\mathrm{N}$ terminal and $\mathrm{C}$ terminal domains. It has been suggested that $\mathrm{N}$ protein is heavily phosphorylated, and this triggers a structural change that enhances the viral RNA [10].

\section{COVID-19 Outbreak and treatment options}

It is the first time that coronavirus has caused a pandemic in humans. However, there are reports on previous outbreaks caused by other $\mathrm{CoV}$ members, such as SARS-CoV and MERS. The current pandemic's causative agent is SARSCoV-2 [11], affecting the human circulatory and respiratory systems. The rapid progression of the disease and its higher transmission rate makes it a severe global health concern. During the early outbreak, a pattern was observed in the infected population, such as fever, body ache, tiredness, difficulty breathing, and lung infection with pneumonia-like symptoms. Currently, more than 185 countries have contacted the disease outbreak [12]. The major challenge in front of the medical healthcare system and scientists is to contain the disease via social distancing and utilizing already available drugs approved by the Food and Drug Administration (FDA), such as Hydroxychloroquine and Remdesivir. There is an urgent need to establish fundamental knowledge and understanding of the hostpathogen interaction to exploit more effective treatment options. Therefore, it is of grave need to understand the pathology of the virus and its target cells, including the immune response to the virus replication and infection. To date, there is no specific approved oral drug to treat novel coronavirus infection. However, a few alternative medicines effectively treat COVID-19 patients, such as Remdesivir, Hydroxychloroquine, chloroquine, Azithromycin, convalescent plasma Tocilizumab, Lopinavir/Ritonavir, Tamiflu, Flavipiravir, Colchicine, Ivermectin, and ACE2 inhibitors, etc. Recent research has shown that Auranofin, an FDA-approved drug for Arthritis treatment, is also very effective in SARS CoV-2 inhibition in human cells $[11,13]$.

A study conducted by Grein et al., with 61 patients from the United States, Europe, and Japan, were administrated 200mg Remdesivir through IV on day one followed by $100 \mathrm{mg}$ for the next nine days. At the end of the study, on March 30, 2020, 36 patients out of 53 showed clinical improvements [14]. On May 1, 2020, the FDA issued an Emergency Use Authorization (EUA) for Remdesivir. That means the FDA has not yet approved Remdesivir for treating COVID-19 patients; however, the drug is easily accessible to doctors for the urgent need of COVID-19 hospitalized patients. On June 1, 2020, Gilead pharmaceutical announced Phase 3 clinical trial results in which the Remdesivir is found to improve the condition in moderate COVID-19 patients. However, still more data and extensive studies are required.

Hydroxychloroquine and chloroquine are FDA-approved drugs used to treat malaria and autoimmune conditions such as Arthritis and Lupus. A very small French population with COVID19 was recruited to establish the efficacy of
Hydroxychloroquine in COVID19 patients. The patients were administrated with $600 \mathrm{mg}$ (three times a day with $200 \mathrm{mg}$ dosage each time) of oral hydroxychloroquine sulfate for 10days. On Day 6, the viral load decreased significantly among the infected group as compared to the control. Subsequently, on March 28, 2020, Hydroxychloroquine is put on the FDA's EUA list [15].

Convalescent plasma (CP) is another treatment method in COVID-19 patients in which blood plasma of infected patients is infused in another COVID-19 patient. A study of 10 adult patients showed that $200 \mathrm{ml}$ of $\mathrm{CP}$ effectively cleared viral load in 7 days. However, larger-scale research and random trials are required before making any final conclusion [16]. Additionally, combinational therapy is also being employed to find the best combination. On May 1, 2020, FDA issued an application of Emergency Investigational New Drug (eIND) for CP as the COVID-19 treatment option. Tocilizumab is a drug used for the treatment of inflammatory conditions like rheumatoid arthritis. Inflammation is a natural response of our immune system against harmful pathogens. Sometimes due to the overactive immune system, inflammations go haywire, causing cytokine storms in which the immune system works against our own body. IL-6 is a major inflammatory cytokine, and Tocilizumab help attenuate inflammation by blocking the IL-6 receptor [17, 18].

Proteases are fundamental for virus replication. Protease inhibitors are used to inhibit SARS-CoV-2 viral replication by inactivating the proteases. Lopinavir/Ritonavir is used in the emergency management plan for COVID-19. Remdesivir is a nucleotide analog previously used in the treatment of the Ebola outbreak in Africa and is currently used in the treatment of COVID-19. Convalescent plasma therapy is also used as a treatment strategy against SARS-CoV-2. Patients recovered from COVID-19 carry the SARS-CoV-2 specific antibody in their blood. Therefore already built antibodies from recovered patients serve as a therapeutic alternative to treat SARS-CoV2 infected patients [19]

Increased cytokine levels and inflammatory response due to the SARS-CoV-2 infection are among the most critical causes of organ damage. Abnormal release of proinflammatory cytokines, mainly IL-6, TNFa, and IFNg, contributes to cytokine release syndrome. Tocilizumab is a monoclonal antibody against the IL-6 receptor is used as a treatment option in severe COVID-19 patients. Corticosteroids are antiinflammatory drugs that are also used for COVID-19 treatment. Published literature suggests thromboembolic manifestations associated with COVID-19. Activation of the coagulation cascade and endothelial injury are indicated as a cause for the development of a prothrombotic state associated with an exaggerated pro-inflammatory response. The use of anticoagulants such as heparin remains an area of conjecture with no definite guidelines of its usage [20].

As of June 17, 2021, according to NIH 
(www.covid19treatmentguidelines.nih.gov), the antiviral drugs that are approved or under consideration for COVID-19 are listed in table: 1

\begin{tabular}{|c|c|}
\hline Treatment category & Treatment options \\
\hline Antiviral drugs & $\begin{array}{c}\text { Remdesivir, Chloroquine or } \\
\text { Hydroxychloroquine with or Without } \\
\text { Azithromycin, Ivermectin, Lopinavir/Ritonavir, } \\
\text { and Other HIV Protease Inhibitors }\end{array}$ \\
\hline Antibody therapy & $\begin{array}{c}\text { Anti-SARS-CoV-2 Monoclonal Antibodies, } \\
\text { Convalescent Plasma, SARS-CoV-2 Specific } \\
\text { Immunoglobulins, IVIG-SARS-CoV-2, }\end{array}$ \\
\hline $\begin{array}{c}\text { Cell-Based } \\
\text { Therapy Under } \\
\text { Evaluation } \\
\text { lung injury and inhibit the cell-mediated } \\
\text { inflammatory response induced by SARS-CoV- } \\
2 .\end{array}$ \\
\hline $\begin{array}{c}\text { Immunomodulators } \\
\text { Colchicine, Corticosteroids, Fluvoxamine, } \\
\text { Interferons, Interleukins-1 and 6 inhibitors, } \\
\text { Kinase inhibitors }\end{array}$ \\
\hline $\begin{array}{c}\text { Anti-thrombotic } \\
\text { therapy }\end{array}$ & Vitamin C, Vitamin D, and Zinc supplements \\
\hline
\end{tabular}

CVD is associated with low-grade chronic inflammation; whether the chronic inflammation synergistically facilitates the virus infection leading to organ failure remains unknown. Secondary organ failure is a major concern in SARS-CoV-2 infected CVD patients. Direct infection of ECs by SARS-CoV-2 has been reported and is associated with a microvascular injury that may be deleterious in prevalent CVD patients [21-23]. In addition to social distancing and isolation measures, careful monitoring of COVID-19 symptoms and frequent evaluation of cardiac and inflammatory biomarkers to identify early signs of cardiac injury can be helpful. Re-optimizing CVD treatments after infection and closer monitoring for post-COVID symptoms can also be useful [24].

ACE-2 has a cardioprotective role, and SARS-CoV-2 internalization into cells has been reported to downregulate ACE-2 expressions[25-29]. In the normal adult lung, ACE-2 is primarily expressed in primary alveolar epithelial type II cells and plays a protective role in the lungs. Surfactant proteins produced by these cells help reduce surface tension and protect alveoli from collapsing[30, 31]. Ang II is the main effector molecule in the RAAS pathway, which is upregulated in many diseases and it's a common target in various cardiovascular disorders [32]. ACE-2 helps inactivate Ang II by converting Ang II to Ang (1-7) [33]. According to a recent study, exogenous administration of recombinant human ACE2 (rhACE-2) can prevent SARS-CoV-2 infection by acting as a decoy. hrACE-2 effectively reduced the infection in cell culture and human blood vessels organoids and kidney organoids. The protective role of hrACE-2 has been reported by different groups in CVDs, rhACE- 2 could be a promising treatment option for CVD patients with COVID-19 infection [34-36]. ACE inhibitors/angiotensin receptor blockers (A.C.E.Is/A.R.Bs) are increasingly used in CVD treatments, and according to studies, they help upregulate ACE-2 expression. The fact that ACE-2 expression could correlate with SARS-CoV-2 susceptibility and intake may predispose CVD patients to increased risk of SARS-CoV-2 infection.
Therefore, the usage of such drugs should be very carefully evaluated in CVD patients [37].

Cardiovascular complications in COVID-19 patients and the role of Endothelial Cells

ACE2 plays an essential role in inhibiting the pathogenic effect of AngII. AngII is a potent inducer of ECs dysfunction, cardiovascular-associated disease, the proliferation of VSMCs, hypertension, and diabetes (Ferrario, Jessup et al. 2005, Tikellis, Bernardi et al. 2011). Functionally, ACE2 promotes the degradation of AngII to Ang-(1-7) and promotes ACEI and AT1R to increase circulating Ang-(1-7) [38]. Ferrario, et al., 2005 and others have established that inhibition of AngII synthesis or its activity by ACEIs (lisinopril) and/or ARBs (losartan) can significantly enhance ACE2 activity [39]. Following the footsteps, a clinical trial is underway to evaluate its effect against COVID-19 (NCT04340557).

At basal conditions, endothelial function in arterial vasculature plays a crucial role in the maintenance of vascular tone by regulating key mechanisms such as adhesion of circulating blood cells, vascular smooth muscle cells (VSMC) growth, and proliferation and inflammation, and immune response. Under normal physiological conditions, ECs maintain basal perfusion determined by cardiac output. Vascular contraction and relaxation in local blood flow are balanced by EC-derived vaso-dilative and vaso-constrictive factors. Nitric oxide (NO) is one of the most critical signaling molecules required to maintain a healthy vasculature. $\mathrm{NO}$ is a potent vasodilator released by EC due to shear stress. NO production results from endothelial nitric oxide synthase (eNOS), in which L-arginine is used as a substrate to produce intracellular cyclic GMP [40]. Compromised NO production in Endothelial dysfunction in the vasculature is profoundly implicated in the pathogenesis of cardiovascular diseases.

A plethora of research has demonstrated that ECs dysfunction is characterized by altered vascular tone, increased inflammatory molecules, and redox imbalance within the blood vessels [41, 42]. Impairment of EC-dependent vasodilation is the hallmark of endothelial dysfunction, responsible for various types of CVD, including diabetes mellitus, hypertension, atherosclerosis, and heart failure [43]. Activation of EC refers to increased expressions of cytokines, chemokines, and adhesion molecules leading to the proinflammatory and prothrombotic microenvironment in the blood vessels.

Underlying CVD complications in COVID-19 is very alarming, especially considering the high number of CVD patients worldwide and in the United States. It is not well understood why CVD enhances COVID-19 infection and severity in infected patients. It is well researched that ECs are essential in maintaining vascular homeostasis and that they play a vital role in the development of CVD. It is still not well known if ECs are also involved in cardiovascular complications in COVID-19 patients. 
. According to Varga, et al., 2020, a kidney transplant 71 years old male patient with coronary artery disease and arterial hypertension died on day eight after COVID 19 infection [44]. Postmortem of the patient's transplanted kidney's electron microscopy showed virus inclusion bodies in ECs of the organ. Histological analysis also showed ECs associated with inflammatory cells and apoptotic bodies in the heart, small intestine, and lungs. Infiltration of mononuclear cells and small congested vessels in the lungs were observed [44]. In another case, a 58-year-old female COVID-19 patient with pre-existing diabetes, arterial hypertension, and obesity developed respiratory failure due to SARS CoV-2 infection. The complications led to multi-organ failure and required renal replacement therapy [44].

Another example is a 69-year-old male patient with hypertension who contracted respiratory failure due to COVID-19 and was put on a mechanical ventilator. Echocardiography showed reduced left ventricular ejection fraction. The patient survived, while histological analysis showed prominent endothelitis of submucosal vessels and apoptotic bodies. This piece of work indicates that SARS CoV-2 binds to the ACE2 expressed by ECs on the host cell membrane, followed by its internalization into the lung, heart, kidneys, brain, and the gut's as these organs predominantly express the ACE2 receptor [44, 45].

Reactive oxygen species (ROS) act as a double-edged sword in CVD and have been implicated in atherosclerosis [46]. Though ROS is required for vascular homeostasis, uncontrolled production has detrimental effects, and ECs maintain this balance of ROS production and oxidative stress (Figure 1). According to Green et al., 2020, the lungs' respiratory distress caused by COVID- 19 quickly extends to the vascular system in the heart, gut, brain, and kidneys in association with ECs dysfunction and fatal blood clotting events [46]. Guan et al., 2020 reported that the mortality rate of patients with CVD-associated diseases such as hypertension was $14.9 \%$, diabetes $7.4 \%$, and coronary heart disease was $2.5 \%$, while hospital stay was approximately 12 days among 1099 patients [47, 48]. Additionally, a large pool of 44672 COVID-19 patients was screened for CVD-associated disorders. About $4.2 \%$ of them had CVD, $12.8 \%$ were hypertensive, and $4.7 \%$ were critically ill. $4.2 \%$ of CVD made around $22.7 \%$ cases of all fatalities reported, with a mean fatality rate being $10.5 \%$. Another study conducted by Huang and Chen et al., 2020 confirms that patients with underlying CVD primes for severe COVID-19 infection in association with severe cytokine storm [17, 18, 48, 49].

ACE2: An advantage or disadvantage in CVD associated SARS CoV-2 infection

Since host ACE2 is the prime target for SARS-CoV-2 infection (the spike protein) and has a stronger binding affinity to human ACE2 $[17,45,50]$ so, manipulating the point of interaction between ACE2 and SARS-CoV-2, or inhibition of ACE2 activity, has gained serious attention and prompted newer dimensions to therapeutic approach (Clinical trials NCT04405999, NCT04353596, NCT04335786, NCT04340557, and NCT04329195). Mechanistically, ACE2 degrade Ang II into Ang-(1-7), while Ang I is degraded to Ang-(1-7) via endopeptidase (NEP), Ang-(1-7) then binds with Mas receptor (Mas-R), facilitating anti-inflammatory, anti-fibrotic response, generate $\mathrm{NO}$ and maintain blood pressure to antagonize the detrimental effect of Ang II [45]. During viral infection, ACE2 binds with SARS-CoV-2, disrupting the traditional pathway responsible for maintaining vascular homeostasis. Internalization of the virus particle in ECs increases viral load to promote cardiovascular complications. Complications associated with CVD patients are administering drugs to inhibit the renin-angiotensinaldosterone system (RAAS or RAS) or the statins [45]. Inhibition of RAAS in hypertensive patients shifts the pathway towards ACE2 mediated Ang-(1-7) generation and further maintained vascular homeostasis. On the other hand, suppression of ACE2 results in the accumulation of Ang II, which stimulates angiotensin II type 1a receptor to increase pulmonary vascular permeability, thus explains the pathology associated with decreased ACE2. While in the case of SARSCoV-2 infection, ACE2 inhibition could be a treatment option, but this option could be unfavorable in CVD patients and those taking ACE2 activators. Atherosclerosis is the most prominent form of CVD and is associated with an increased level of Ang II. A study by Tesanovic et al., 2010, establishes that Ang-(1-7) infusion in ApoE-/- mice fed with a high-fat diet can significantly improve ECs function, which confers atheroprotection via restoration of available NO, while inhibition of RAS can prevent atherosclerosis in ApoE/Ace2 doubleKO mice [51, 52]. A combination of ACE inhibitors (ACEIs) to enhance ACE2 activity may help cardiovascular patients suffering from COVID-19. Owing to the critical role played by the ACE2 receptor, various clinical trials are focused on finding the alternative to overcome the ACE 2 pathway [17, 49]. Some of the critical clinical trials are Bromhexine Hydrochloride and valsartan; some recombinant proteins used such as - Recombinant human angiotensinconverting enzyme 2 (rhACE2) - APN01, Recombinant Bacterial ACE2 receptors -like enzyme of B38-CAP.

\section{DISCUSSION}

Researchers worldwide explore various avenues to establish the cure, decrease mortality rate, and minimize hospital stay due to SARS CoV-2. Having pre-existing CVD in COVID-19 patients results in severe complications. It makes it more difficult for clinicians to establish a fine-tune between available drugs and treatment methodology to save a patient's life. Maintaining a proper clinical record and standard procedure utilization is crucial to establish a generalized treatment strategy that holds the key to a successful drug trial. The discrepancy in research methodology and negligence to recognize genetic and geographical variations have been a roadblock to achieving consensus for the COVID19 treatment strategy. 
Approximately 735,000 Americans suffer a heart attack every year, 525,000 undergo a first heart attack, and 210,000 have already had a heart attack [39]. ECs dysfunction or injury was first proposed more than 20 years ago, and then several scientific works of literature came out in support of this concept [28]. There is extensive evidence that ECs dysfunction is of primary importance in the pathogenesis of atherosclerosis and lesion formation [53]. EC is the first line of defense between various risk factors and vascular disease. EC dysfunction is the starting stage of atherosclerosis and an important prognostic marker of CVD. Since ECs are in direct contact with blood flow and serves as a barrier in the vascular system, it is susceptible to any change in the blood vessels. $\mathrm{EC}$ is the first line of defense between various risk factors and vascular disease. Endothelial cells in the brain are known as brain endothelial microvascular cells. A recent study showed that SARS-COV2 could infect the central nervous system in mice brain and cause neuroinflammation and encephalitis [54]. As the number of research concerning SARS-CoV2 grows, it is expected to unfold many more undetermined factors. To better understand the cause and effect of CVDCOVID-19 infection, we need to explore cardiovascular complications in specific conditions, along with the identification of disease models. Mesenchymal stem cells are multipotent adult stem cells found in most human tissues. These cells can regenerate and differentiate into multiple tissue types. Therefore, Mesenchymal stem cells could have an enormous scope in regenerative medicine. Moreover, they lack ACE2 receptors to which SARS-CoV-2 binds for entry into cells; these cells are resistant to infection making them a promising target against SARS-CoV-2 [55, 56].

Figure 1:

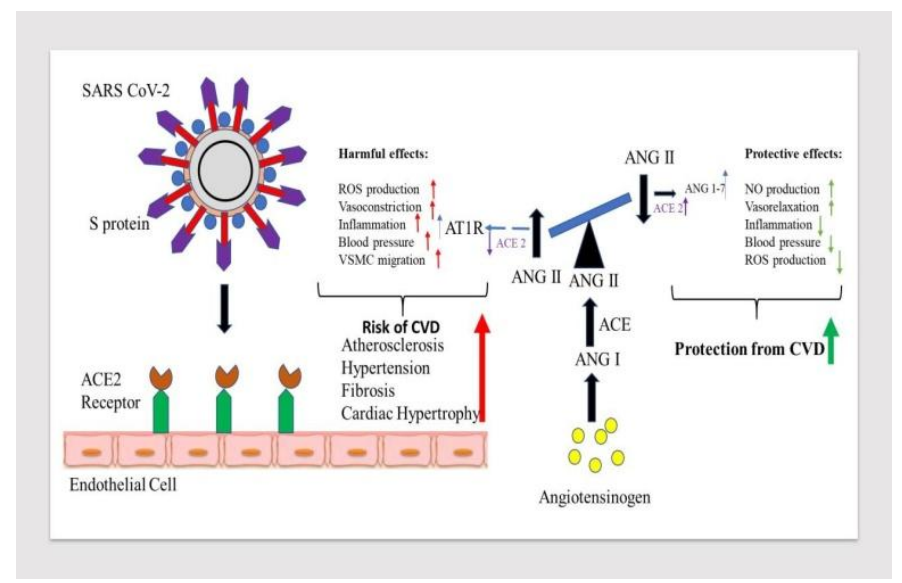

Figure 1: The SARS CoV-2 binds to ACE2 receptor on endothelial cells by its $\mathrm{S}$ protein. ACE2 has a cardioprotective effect. On the contrary, increased ANG II in the absence of ACE2 increases the risk of CVD. SARS CoV-2, Severe Acute Respiratory Syndrome Coronavirus 2; S protein, Spike protein; ANG, Angiotensin; AT1R, Angiotensin 1 receptor; NO, nitric oxide; ROS, reactive oxygen species.

\section{REFERENCE}

[1] Naghavi, M., et al., Causes of Death C: Global, regional, and national age-sex specific all-cause and cause-specific mortality for 240 causes of death, 1990-2013: a systematic analysis for the Global Burden of Disease Study 2013. 2015.

[2] Mitra, S., et al., Oxidized low-density lipoprotein and atherosclerosis implications in antioxidant therapy. The American journal of the medical sciences, 2011. 342(2): p. 135-142.

[3] Daiber, A., et al., Targeting vascular (endothelial) dysfunction. British journal of pharmacology, 2017. 174(12): p. 1591-1619.

[4] BURNSTOCK, G., Release of vasoactive substances from endothelial cells by shear stress and purinergic mechanosensory transduction. The Journal of Anatomy, 1999. 194(3): p. 335-342.

[5] Gheibi, S., et al., Regulation of vascular tone homeostasis by $\mathrm{NO}$ and H2S: Implications in hypertension. Biochemical pharmacology, 2018. 149: p. 42-59.

[6] Park, K.-H. and W.J. Park, Endothelial dysfunction: clinical implications in cardiovascular disease and therapeutic approaches. Journal of Korean medical science, 2015. 30(9): p. 1213-1225.

[7] Gimbrone Jr, M.A. and G. García-Cardeña, Endothelial cell dysfunction and the pathobiology of atherosclerosis. Circulation research, 2016. 118(4): p. 620-636.

[8] Nieto-Torres, J.L., et al., Severe acute respiratory syndrome coronavirus envelope protein ion channel activity promotes virus fitness and pathogenesis. PLoS pathogens, 2014. 10(5).

[9] Seah, I., X. Su, and G. Lingam, Revisiting the dangers of the coronavirus in the ophthalmology practice. 2020, Nature Publishing Group.

[10] Stohlman, S.A. and M. Lai, Phosphoproteins of murine hepatitis viruses. Journal of virology, 1979. 32(2): p. 672-675.

[11] Rothan, H.A. and S.N. Byrareddy, The epidemiology and pathogenesis of coronavirus disease (COVID-19) outbreak. Journal of autoimmunity, 2020: p. 102433.

[12] Organization, W.H., Coronavirus disease 2019 (COVID-19): situation report, 82. 2020.

[13] Rothan, H.A., et al., The FDA-approved gold drug Auranofin inhibits novel coronavirus (SARS-COV-2) replication and attenuates inflammation in human cells. Virology, 2020.

[14] Grein, J., et al., Compassionate use of remdesivir for patients with severe Covid-19. New England Journal of Medicine, 2020. 382(24): p. 2327-2336.

[15] Wang, M., et al., Remdesivir and chloroquine effectively inhibit the recently emerged novel coronavirus (2019- $n \mathrm{CoV})$ in vitro. Cell research, 2020. 30(3): p. 269-271.

[16] Duan, K., et al., Effectiveness of convalescent plasma therapy in severe COVID-19 patients. Proceedings of the National Academy of Sciences, 2020. 117(17): p. 9490-9496.

[17] Huang, C., et al., Clinical features of patients infected with 2019 novel coronavirus in Wuhan, China. The lancet, 2020. 395(10223): p. 497-506.

[18] Moore, J.B. and C.H. June, Cytokine release syndrome in severe COVID-19. Science, 2020. 368(6490): p. 473-474.

[19] Stasi, C., et al., Treatment for COVID-19: An overview. 2020: p. 173644.

[20] Chandra, A., et al., Anticoagulation in COVID-19: current concepts and controversies. 2021.

[21] Clerkin, K.J., et al., COVID-19 and cardiovascular disease. 2020. 141(20): p. 1648-1655.

[22] Driggin, E., et al., Cardiovascular considerations for patients, health care workers, and health systems during the COVID-19 pandemic. 2020. 75(18): p. 2352-2371.

[23] Pothineni, N.V.K., et al., Infections, atherosclerosis, and coronary heart disease. 2017. 38(43): p. 3195-3201.

[24] Chatterjee, N.A. and R.K. Cheng, Cardiovascular disease and COVID-19: implications for prevention, surveillance and treatment. 2020, BMJ Publishing Group Ltd and British Cardiovascular Society.

[25] Oudit, G., et al., SARS-coronavirus modulation of myocardial ACE2 expression and inflammation in patients with SARS. 2009. 
39(7): p. 618-625.

[26] Kuba, K., et al., A crucial role of angiotensin converting enzyme 2 (ACE2) in SARS coronavirus-induced lung injury. 2005. 11(8): p. 875-879.

[27] Li, W., et al., Angiotensin-converting enzyme 2 is a functional receptor for the SARS coronavirus. 2003. 426(6965): p. 450-454.

[28] Thomas, M.C., et al., Genetic Ace2 deficiency accentuates vascular inflammation and atherosclerosis in the ApoE knockout mouse. 2010. 107(7): p. 888-897.

[29] Jiang, F., et al., Angiotensin-converting enzyme 2 and angiotensin 1-7: novel therapeutic targets. 2014. 11(7): p. 413-426.

[30] Sungnak, W., et al., SARS-CoV-2 entry factors are highly expressed in nasal epithelial cells together with innate immune genes. 2020. 26(5): p. 681-687.

[31] Qi, F., et al., Single cell RNA sequencing of 13 human tissues identify cell types and receptors of human coronaviruses. 2020. 526(1): p. 135-140.

[32] Vaduganathan, M., et al., Renin-angiotensin-aldosterone system inhibitors in patients with Covid-19. 2020. 382(17): p. 1653-1659.

[33] Turner, A.J., J.A. Hiscox, and N.M.J.T.i.p.s. Hooper, ACE2: from vasopeptidase to SARS virus receptor. 2004. 25(6): p. 291-294.

[34] Monteil, V., et al., Inhibition of SARS-CoV-2 infections in engineered human tissues using clinical-grade soluble human ACE2. 2020. 181(4): p. 905-913. e7.

[35] Haschke, M., et al., Pharmacokinetics and pharmacodynamics of recombinant human angiotensin-converting enzyme 2 in healthy human subjects. 2013. 52(9): p. 783-792.

[36] Khan, A., et al., A pilot clinical trial of recombinant human angiotensin-converting enzyme 2 in acute respiratory distress syndrome. 2017. 21(1): p. 1-9.

[37] Lia, Y., et al., Expert recommendations for management and treatment of cardiovascular diseases under the epidemic situation of novel coronavirus pneumonia in Hubei province. 2020. 36(3): p. 1-3.

[38] Stanziola, L., L.J. Greene, and R.A. Santos, Effect of chronic angiotensin converting enzyme inhibition on angiotensin $I$ and bradykinin metabolism in rats. American journal of hypertension, 1999. 12(10): p. 1021-1029.

[39] Ferrario, C.M., et al., Effect of angiotensin-converting enzyme inhibition and angiotensin II receptor blockers on cardiac angiotensin-converting enzyme 2. Circulation, 2005. 111(20): p. 2605-2610.

[40] Zhao, Y., P.M. Vanhoutte, and S.W.J.J.o.p.s. Leung, Vascular nitric oxide: Beyond eNOS. 2015. 129(2): p. 83-94.

[41] Boulanger, C.M.J.A., thrombosis, and v. biology, Endothelium. 2016. 36(4): p. e26-e31.

[42] Ooi, B.K., et al., The role of natural products in targeting cardiovascular diseases via Nrf2 pathway: novel molecular mechanisms and therapeutic approaches. 2018. 9: p. 1308.

[43] Suryavanshi, S.V. and Y.A.J.F.i.p. Kulkarni, $N F-\kappa \beta$ : a potential target in the management of vascular complications of diabetes. 2017. 8: p. 798.

[44] Varga, Z., et al., Endothelial cell infection and endotheliitis in COVID-19. The Lancet, 2020. 395(10234): p. 1417-1418.

[45] South, A.M., D.I. Diz, and M.C. Chappell, COVID-19, ACE2, and the cardiovascular consequences. American Journal of Physiology-Heart and Circulatory Physiology, 2020.

[46] Green, S.J., Covid-19 accelerates endothelial dysfunction and nitric oxide deficiency. Microbes and Infection, 2020.

[47] Guan, W., et al., China Medical Treatment Expert Group for Covid-19. Clinical characteristics of coronavirus disease, 2019: p. 1708-1720.

[48] Madjid, M., et al., Potential effects of coronaviruses on the cardiovascular system: a review. JAMA cardiology, 2020.

[49] Chen, N., et al., Epidemiological and clinical characteristics of 99 cases of 2019 novel coronavirus pneumonia in Wuhan, China: a descriptive study. The Lancet, 2020. 395(10223): p. 507-513.

[50] Li, F., et al., Structure of SARS coronavirus spike receptor-binding domain complexed with receptor. Science, 2005. 309(5742): p. 1864-1868.

[51] Tesanovic, S., et al., Vasoprotective and atheroprotective effects of angiotensin (1-7) in apolipoprotein E-deficient mice. Arteriosclerosis, thrombosis, and vascular biology, 2010. 30(8): p. 1606-1613.

[52] Thomas, M.C., et al., Genetic Ace2 deficiency accentuates vascular inflammation and atherosclerosis in the ApoE knockout mouse. Circulation research, 2010. 107(7): p. 888-897.

[53] Cui, J., F. Li, and Z.-L. Shi, Origin and evolution of pathogenic coronaviruses. Nature reviews Microbiology, 2019. 17(3): p. 181192.

[54] Kumari, P., et al., Neuroinvasion and encephalitis following intranasal inoculation of SARS-CoV-2 in K18-hACE2 mice. Viruses, 2021. 13(1): p. 132.

[55] Lukomska, B., et al., Challenges and controversies in human mesenchymal stem cell therapy. 2019. 2019.

[56] Shetty, A.K.J.A. and disease, Mesenchymal stem cell infusion shows promise for combating coronavirus (COVID-19)-induced pneumonia. 2020. 11(2): p. 462. 